\title{
Ferroelectric nanofibers with an embedded optically nonlinear benzothiazole derivative
}

\author{
Rosa M. F. Baptista, ${ }^{a}$ Dmitry Isakov, ${ }^{b}$ M. Manuela M. Raposo,${ }^{* a}$ Michael Belsley, ${ }^{b}$ Igor Bdikin, ${ }^{c}$ \\ Andrei L. Kholkin, ${ }^{d, e}$ Susana P. G. Costa, ${ }^{a}$ and Etelvina de Matos Gomes* ${ }^{b}$
}

\begin{abstract}
${ }^{a}$ Center of Chemistry, University of Minho, Campus de Gualtar, 4710-057 Braga, Portugal. Fax: 00351 253604382; Tel:00351253 604381; E-mail: mfox@quimica.uminho.pt; rosa_batista@quimica.uminho.pt; spc@quimica.uminho.pt.

${ }^{b}$ Center of Physics, University of Minho, Campus de Gualtar, 4710-057 Braga, Portugal. Fax: 00351 253604061; Tel: 00351 253604339; E-mail: emg@fisica.uminho.pt;dmitry@fisica.uminho.pt; belsley@fisica.uminho.pt.

${ }^{c}$ Department of Mechanical Engineering and TEMA, University of Aveiro, 3810-193 Aveiro, Portugal; E-mail: bdikin@ua.pt

${ }^{d}$ Department of Ceramics and Glass Engineering \& CICECO, University of Aveiro, 3810-193 Aveiro, Portugal; Tel: 00351 23424702; E-mail: kholkin@ua.pt

${ }^{e}$ Institute of Natural Sciences, Ural Federal University, 620000 Ekaterinburg, Russia; Tel: 007 3432617436, E-mail: kholkin@ua.pt.

*corresponding author:mfox@quimica.uminho.pt;emg@fisica.uminho.pt;
\end{abstract}

Keywords: benzothiazole; nonlinear optics (NLO), nanofibers; PLLA fibers; piezoelectric material.

\begin{abstract}
We report measurements of the molecular first hyperpolarizability, thermal stability, photophysical, piezoelectric and ferroelectric properties of a benzothiazole derivative bearing an arylthiophene $\pi$-conjugated bridge both in solution and when embedded into a poly (L-lactic acid) (PLLA) matrix in the form of electrospun fibers with an average diameter of roughly $500 \mathrm{~nm}$. The embedded nanocrystalline phenylthienyl-benzothiazole derivative, with crystal sizes of about $1.4 \mathrm{~nm}$ resulted in a good piezoelectric response from these functionalized electrospun fibers, indicative of a polar crystalline structure.
\end{abstract}

\section{Introduction}

Benzothiazole derivatives have attracted significant attention recently due to their desirable optical (linear and nonlinear) properties. They have been shown to possess high quantum fluorescence yields and excellent molecular hyperpolarizabilities which can be tuned by functionalization of the azole skeleton with 5-membered heterocycles (pyrrole, thiophene) at position 2. This modification creates the potential for numerous innovative applications of these $\pi$-conjugated systems as ligands for phosphorescent coordination complexes including transition metals, antennas for lanthanoid elements ( $\mathrm{Li}$ et al. 2012; Kuwabara et al. 2012; Wang et al. 2011; Liu et al. 2011), in nonlinear optics (e.g. second harmonic generators (SHG) or two photon absorbers (TPA)) (Miller et al. 1994, Varanasi et al. 1996; Albert et al. 1997; Breitung et al. 2000; Hrobarik et al. 2004; Cui et al. 2006; Coe et al. 2006; Razus et al. 2007; Jin et al. 2009; Hrobárik et al. 2010; Hrobáriková et al. 2010; Hrobarik et al. 2011; Kariduraganavar et al. 2011), as well as fluorescent dopants in the fabrication of organic light emitting devices (OLEDs) (Tao et al. 2011, Mishra et al. 2009; Mabrouk et al. 2010; Fu et al. 2009a; Fu et al. 2009b; Xu et al. 2007; Zhang et al. 2001). Recently we reported the synthesis and characterization of the optical properties of novel benzothiazole derivatives bearing bithiophene, arylthiophene and (bi)thienylpyrrole $\pi$-bridges in solution (Costa et al. 1997, Batista et al. 2004; Costa et al. 2006; Batista et al. 2007; Pina et al. 2010; Raposo et al. 2011a; Raposo et al. 2011b; Raposo et al. 2011c; Coelho et al. 2012a; Coelho et al. 2012b) in liquid crystals (GarciaAmorós et al. 2013; Garcia-Amorós et al. 2014), and in PMMA or Zeonex matrixes (Coelho et al. 2013; Pina et al. 2007). Our theoretical studies (Pina et al. 2010; Raposo et al. 2011a) were focused on the optimization of the groundstate molecular geometries guided by the results of density functional theory (DFT) calculations for donor-acceptor systems in which the benzothiazole heterocycle played the role of an acceptor group. The experimental studies were mainly focused on the nonlinear optical, photochromic, emissive and OLEDs properties of these compounds. Evaluation of the SHG in solution, using hyper-Rayleigh 
scattering technique, showed that benzothiazole derivatives functionalized with arylthiophene, bithiophene or thienylpyrrole $\pi$-bridges have high molecular nonlinearities with first hyperpolarizability values that are 20-33 times higher than that of the well-known $p$ nitroaniline ( $p$ NA) molecule (Batista et al. 2004; Costa et al. 2006; Raposo et al. 2011a; Raposo et al. 2011b).

The present work was motivated by an interest to extend the possible applications of benzothiazole derivatives bearing thiophenic bridges. A significant limitation in this regard has been the inability to grow crystals with good quality and size compatible for practical applications. One possibility of overcoming this problem is to incorporate the compound inside a host polymer matrix using a nanomanufacturing process. Our approach consists in embedding functional nanocrystalline structures inside aligned polymer nanofibers by the electrospinning technique (Isakov et al. 2010a; Isakov et al. 2011a, Isakov et al. 2011b; Isakov et al. 2012)

Indeed, some of us recently reported evidence of strong polar properties of chromophores embedded in polymer nanofibers. Significant second harmonic generation and piezoelectric responses were obtained in nanofibers produced by the electrospinning technique. Due to the strong processing electric field and mechanical stretching applied during the drawing of the fibers, the electrospinning process can produce anisotropic piezoelectric nanofibers with enhanced properties (Li et al. 2003; Persano et al. 2013; Isakov et al. 2011b).

Here we report the molecular linear and nonlinear optical properties, of a benzothiazole derivative bearing an phenylthiophene bridge (BZT 1), previously synthesised by some of us (Costa et al. 1997). We also report the thermal stability as well as the piezoelectric response of electrospun PLLA nanofibers with embedded crystalline BZT 1. To the best of our knowledge this is the first time that the nonlinear optical properties in solution and in PLLA fibers are reported.

\section{Experimental}

\section{Instruments}

Fluorescence spectra were collected using a FluoroMax-4 spectrofluorometer. UV-visible absorption spectra (200$700 \mathrm{~nm}$ ) were obtained using a Shimadzu UV/2501PC spectrophotometer. The fluorescence quantum yield was measured using a solution of 9,10-diphenylanthracene in ethanol as standard $\left(\varphi_{\mathrm{F}}=0.95\right)$ (Morris et al. 1976). Thermogravimetric analysis of samples was carried out using a TGA instrument model Q500 from TA Instruments, under high purity nitrogen supplied at a constant $50 \mathrm{~mL} \mathrm{~min}{ }^{-1}$ flow rate. All samples were subjected to a $20{ }^{\circ} \mathrm{C} \min ^{-1}$ heating rate and were characterized between 25 and $500{ }^{\circ} \mathrm{C}$.

Nonlinear optical measurements using the hyper-Rayleigh scattering (HRS) method
Hyper-Rayleigh scattering (HRS) was used to measure the first hyperpolarizability $\beta$ of response of the molecules studied. The experimental set-up for hyper-Rayleigh measurements has been previously described in detail (Raposo et al. 2011a). The incident laser beam came from a Q-switched Nd:YAG laser operating at a $10 \mathrm{~Hz}$ repetition rate with approximately $5 \mathrm{~mJ}$ of energy per pulse and a pulse duration (FWHM) close to $12 \mathrm{~ns}$ at the fundamental wavelength of $1064 \mathrm{~nm}$. The incident beam was weakly focused (beam diameter $\sim 0.5 \mathrm{~mm}$ ) into the solution contained in a $5 \mathrm{~cm}$ long cuvette.

The hyper-Rayleigh signal was normalized at each pulse using the second harmonic signal from a $1 \mathrm{~mm}$ quartz plate to compensate for fluctuations in the temporal profile of the laser pulses due to longitudinal mode beating. Dioxane was used as a solvent, and the $\beta$ values were calibrated using a reference solution of $p \mathrm{NA}$ also dissolved in dioxane at a concentration of $1 \times 10^{-2} \mathrm{~mol} \mathrm{dm}^{-3}$ (external reference method). All solutions were filtered $(0.2 \mu \mathrm{m}$ porosity) to avoid spurious signals from suspended impurities.

We took particular care to avoid reporting artificially high hyperpolarizibilities due to a possible contamination of the hyper Rayleigh signal by molecular fluorescence near 532 $\mathrm{nm}$ by employing two different narrow band interference filters (CVI model F1.5-532-4) and "wide" (CVI model F03-532-4) with passbands of $1.29 \mathrm{~nm}$ and $2.18 \mathrm{~nm}$ (full width at half maximum) respectively. This allows us to estimate the amount of hyper-Rayleigh and fluorescence signal (Raposo et al. 2011a).

\section{Local piezoelectric measurements using PFM}

The piezoresponse force microscopy (PFM) signal was investigated using a commercial atomic force microscopy (AFM) setup where the conducting tip is used both to excite the piezoelectric vibrations and to study the vibrational response via an additional lock-in amplifier connected to the photodiode and synchronized with the driving voltage. PFM images were obtained using a Ntegra Aura, NT-MDT instrument where ac and dc voltages were applied between the counter electrode and the conductive tip. The PFM micrograph was obtained in the PFM mode (frequency of ac voltage was $50 \mathrm{kHz}$ and amplitude was 1 $\mathrm{V}$ peak-to-peak). During scanning, the vertical signal from photodiode was amplified by a Stanford Research SR830A lock-in amplifier and imaged using an auxiliary channel of the microscope. The hysteresis loop was acquired after each dc voltage step was applied to the top of the single BZT 1 fiber.

\section{Results and discussion}

\section{Electrospinning of nanofibers}

The benzothiazole derivative (BZT 1, Figure 1) functionalized with an arylthiophene $\pi$-conjugated bridge was synthesized through a previously described synthetic methodology (Costa et al. 1997) by reaction of $o$ aminobenzenethiol with 5-phenylthiophene-2- 
carbaldehyde, in DMSO at $120{ }^{\circ} \mathrm{C}$ for $30-60 \mathrm{~min}$. The reaction is initiated by the formation of the corresponding imine that cyclises spontaneously, yielding the benzothiazoline, which is oxidised to the benzothiazole, in the presence of DMSO.

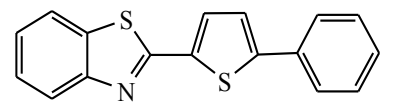

Fig. 1. Molecular structure of phenylthienyl-benzothiazole (BZT) 1

Several attempts were made to obtain appropriate BZT crystals for structure determination by single crystal X-ray diffraction and study of crystalline physico-chemical properties. However this was not possible due to fragility of the obtained crystals which were not suitable for structural work. However they could be used in a crystalline form by using an approach that combines nanomanufacturing process and molecular engineering (Isakov et al. 2011b). The approach consists in fabrication of mesocrystalline structures of aligned polymer nanofibers with embedded functional materials by the electrospinning technique.

The compound has been incorporated in poly (L-lactic acid) (PLLA) fiber host by the conventional electrospinning technique. PLLA was chosen as a host polymer matrix as it permits the use of an appropriate solvent for both polymer and BZT 1 compound. To prepare the solution, $\left(0.4 \mathrm{~g}, 4.71 \times 10^{-6} \mathrm{~mol}\right)$ of poly(Llactic acid) (PLLA, $M_{w} \sim 85000$, purchased from Aldrich and used as received) was dissolved in $4 \mathrm{ml}$ of chloroform and mixed with $1.0 \mathrm{ml}$ of dimethylformamide (DMF, both purchased from Aldrich) and (0.1 g, $\left.3.41 \times 10^{-4} \mathrm{~mol}\right)$ of BZT 1. The obtained solution was stirred for several hours under ambient conditions prior to performing the electrospinning. The precursor solutions were loaded into a syringe with its needle connected to the anode of a high voltage power supply (Spellmann CZE2000). The electric potential and distance between needle and collector were $12-15 \mathrm{kV}$ and 10-12 $\mathrm{cm}$ respectively. The obtained fibers are smooth and uniform with an average diameter of roughly $500 \mathrm{~nm}$.

(a)

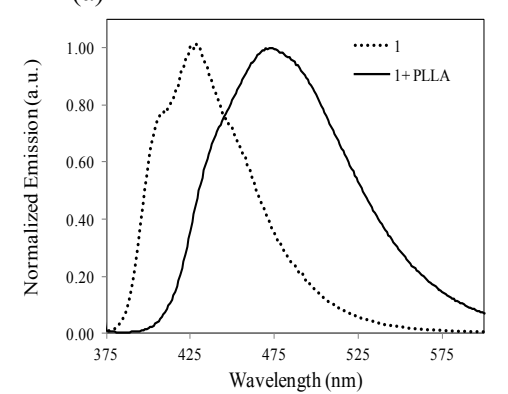

(b)

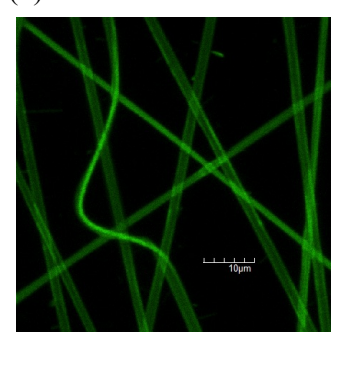

wavelength range of $430-470 \mathrm{~nm}$ following excitation at a wavelength of $405 \mathrm{~nm}$.

\section{Optical and thermal properties}

The photochemical and photophysical properties of compounds such as BZT 1 can be attributed to the presence of two different chromophores (benzothiazole and arylthiophene). The absorption and emission spectra of BZT were measured in absolute ethanol $\left(10^{-4}\right.$ and $10^{-6} \mathrm{M}$ solutions respectively). Electronic absorption spectra of the BZT in absolute ethanol show an intense charge-transfer absorption band in the UV/Vis region $\left(\lambda_{\max }=362 \mathrm{~nm}\right.$; Log $\left.\varepsilon=4.43, \lambda_{\text {emi }}=430 \mathrm{~nm}\right)$.

The relative fluorescence quantum yield was determined using a $10^{-6} \mathrm{M}$ solution of 9,10-diphenylanthracene in ethanol as a standard $\left(\varphi_{\mathrm{F}}=0.95\right)$ (Morris et al. 1976). The BZT compound displays a high fluorescence quantum yield of 0.58 (Table 1, Fig. 2a). The absorption and emission of BZT 1 in PLLA nanofibers exhibit a red shift of 32 and $34 \mathrm{~nm}$ relative to the corresponding data in ethanol (Fig. 2). Red-shifts can indicate a more extended conjugation, in turn attributable to stretched molecular conformations along the fiber longitudinal axis. A higher density related to a more effective molecular packing at nanoscale can also lead to some self absorption of the emitted fluorescence, also contributing to a red shift of the observed emission.

The molecular first hyperpolarizability $\beta$ of BZT 1 was measured by hyper-Rayleigh scattering (HRS) method (Raposo et al. 2011a), at a fundamental wavelength of $1064 \mathrm{~nm}$ of a Q-switched Nd:YAG laser. Dioxane was used as the solvent, and the $\beta$ values were measured against a reference solution of $p$ NA (Kaatz and Shelton 1996; Reis 2006) in order to obtain quantitative values, while care was taken to properly account for possible fluorescence of the dyes (see experimental section for more details). The static hyperpolarizability $\beta_{0}$ value (Oudar 1977; Oudar and Chemla 1977; Zyss and Oudar 1982) was estimated using a simple two-level model neglecting damping. They are therefore only indicative and should be treated with caution (Table 1). The nonlinear optical chromophore BZT 1 exhibits a molecular nonlinearity $\beta$ of $51 \times 10^{-30}$ esu which is 3 times higher than that of the well-known $p$ NA molecule for an incident laser wavelength of $1064 \mathrm{~nm}$ (the corresponding $\beta_{0}$ values are 2.6 times higher than that of $p \mathrm{NA}$ ).

It is well known that, not only a high hyperpolarizability but also a good thermal stability is critical for practical application of organic materials. Consequently, the thermal stability of BZT 1 was evaluated by thermogravimetric analysis. BZT 1 exhibits a high decomposition temperature $\left(\mathrm{T}_{d}=345^{\circ} \mathrm{C}\right)$, measured at a heating rate of $20{ }^{\circ} \mathrm{C} \mathrm{min}{ }^{-1}$ under a nitrogen atmosphere (Fig. 3).

Fig. 2 (a) Normalized emission spectra of BZT nanofibers and the pure compound in absolute ethanol; (b) single-photon fluorescence image $(50 \times 50 \mu \mathrm{m})$ of BZT 1 nanofibers. The fluorescence was observed in the 
Table 1. UV-vis absorption and emission data in ethanol, $\beta$ and $\beta_{0}$ values for BZT 1 in dioxane and BZT 1+ PLLA.

\begin{tabular}{|c|c|c|c|c|c|c|c|}
\hline \multirow[b]{2}{*}{ Cpd. } & \multicolumn{2}{|c|}{ Absorption } & \multicolumn{3}{|c|}{ Emission } & \multirow{2}{*}{$\begin{array}{c}\beta^{\mathrm{a}} \\
\left(10^{-30}\right. \\
\text { esu })\end{array}$} & \multirow{2}{*}{$\begin{array}{c}\beta_{0}{ }^{\mathrm{b}} \\
\left(10^{-30}\right. \\
\mathrm{esu}) \\
\end{array}$} \\
\hline & $\begin{array}{c}\lambda_{\max } \\
(\mathrm{nm})\end{array}$ & $\log \varepsilon$ & $\begin{array}{l}\lambda_{\max } \\
(\mathrm{nm})\end{array}$ & $\begin{array}{c}\text { Stokes' } \\
\text { shift (nm) }\end{array}$ & $\phi$ & & \\
\hline BZT 1 & 362 & 4.43 & 430 & 68 & 0.58 & 51 & 22 \\
\hline PLLA & 330 & - & 425 & 95 & - & - & - \\
\hline $1+$ PLLA & 394 & - & 464 & 70 & - & - & - \\
\hline$p \mathrm{NA}$ & 352 & - & - & - & - & 16.9 & 8.5 \\
\hline
\end{tabular}

a All the compounds are transparent at the $1064 \mathrm{~nm}$ fundamental wavelength.

${ }^{\mathrm{b}}$ Data corrected for resonance enhancement at $532 \mathrm{~nm}$ using the twolevel model with $\beta_{0}=\beta\left[1-\left(\lambda_{\max } / 1064\right)^{2}\right]\left[1-\left(\lambda_{\max } / 532\right)^{2}\right]$; damping factors not included at $1064 \mathrm{~nm}$ (Oudar 1977; Oudar and Chemla 1977; Zyss and Oudar 1982).

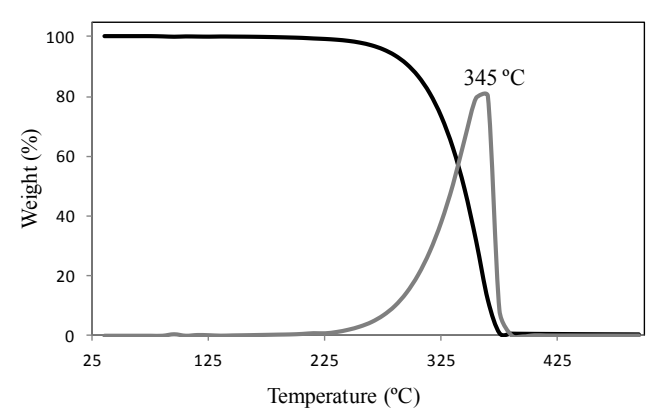

Fig. 3 Thermal analysis data for BZT 1 through TGA recorded under a nitrogen atmosphere, measured at a heating rate of $20{ }^{\circ} \mathrm{C} \mathrm{min}{ }^{-1}$.

\section{Local piezoelectric and ferroelectric properties}

For practical applications of organic materials with high molecular hyperpolarizability it is important that molecules crystallize in a non-centrosymmetric unit cell packing, a necessary condition for observing crystalline polar phenomena such as second harmonic generation, piezoelectricity and pyroelectricity. Piezoresponse force microscopy (PFM) (Balke et al. 2009) was employed to investigate the local piezoelectric properties of BZT 1 nanofibers. The PFM technique is based on the detection of an electromechanical response of the sample to an applied electric voltage and is described elsewhere (Isakov et al. $2010 \mathrm{~b}$ ). The piezo-response contrast is obtained in the form of mixed signal $A \cos \phi$ (where $A$ is the amplitude and $\phi$ is the phase shift between driving and detected signal) providing information on the polarization direction.

Figure 4 presents the PFM images obtained from a single BZT nanofiber deposited on a conductive substrate. The first image shows the as-electrospun nanofiber before any external polarization field was applied. In the next two images (Fig. 4b-c) the out-of-plane PFM contrast is shown after applying $+100 \mathrm{~V}$ (image b) and then $-100 \mathrm{~V} \mathrm{DC}$ voltage (image $\mathrm{c}$ ) to the conductive cantilever tip during 10 s. The strong contrast observed in the PFM images is caused by an induced deformation in response to the applied "read" ac electric field and represents the polarization vector $P$ components of the fiber.
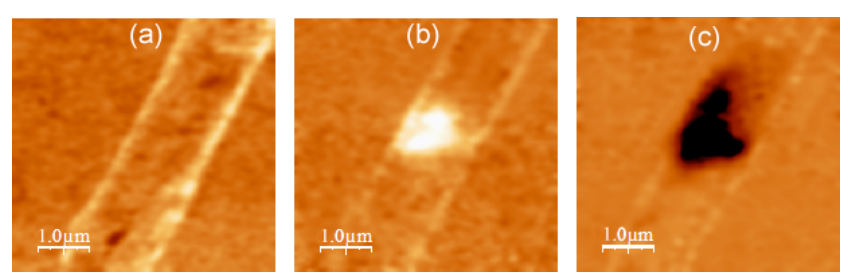

Fig. 4 Vertical PFM images observed in an individual BZT nanofiber: a) as-electrospun nanofibers (before poling); b) PFM image after applying a voltage of $+100 \mathrm{~V}$ at the fixed tip during $10 \mathrm{~s}$; c) image after applying a voltage of $-100 \mathrm{~V}$.

The contrast on the PFM image is roughly proportional to the longitudinal piezoelectric coefficient and is determined by the projection of the polarization vector normal to the substrate. The phase $\phi$ of the signal depends on the orientation of the polarization, thus the "bright" contrasts for the measured $A \cos \phi$ signal suggest that the polarization head is terminated at the substrate plane while the dark contrasts correspond to the polarization pointing downward to the surface.

Figure 5 presents nested piezoresponse hysteresis loops measured on the same BZT 1-nanofiber. The local longitudinal remnant piezo-response signal was measured after the application of continuously increasing DC voltage pulses to the conductive tip fixed on the surface of the BZT 1 fiber. The existence of two switchable polarization states is thus confirmed by the PFM measurements in a dynamic regime in which the bias is continuously swept between positive and negative values. After the first hysteresis, the closed loops were repeatedly acquired, indicating that the polarization switching is quite robust in this material. The corresponding effective piezoelectric coefficient (remnant value) was estimated to be $\mathrm{d}_{33} \approx 20 \mathrm{pm} / \mathrm{V}$. This value is comparable to the piezoelectric coefficient of a poled poly(vinydelene fluoride) film and dabcoHReO4 nanofibers (Isakov et al. 2014). The switching of polarization definitely indicates the ferroelectric nature and an acentric crystalline structure for BZT 1, as ferroelectricity is only possible in polar crystallographic point groups lacking a center of inversion (Nye 2000). 


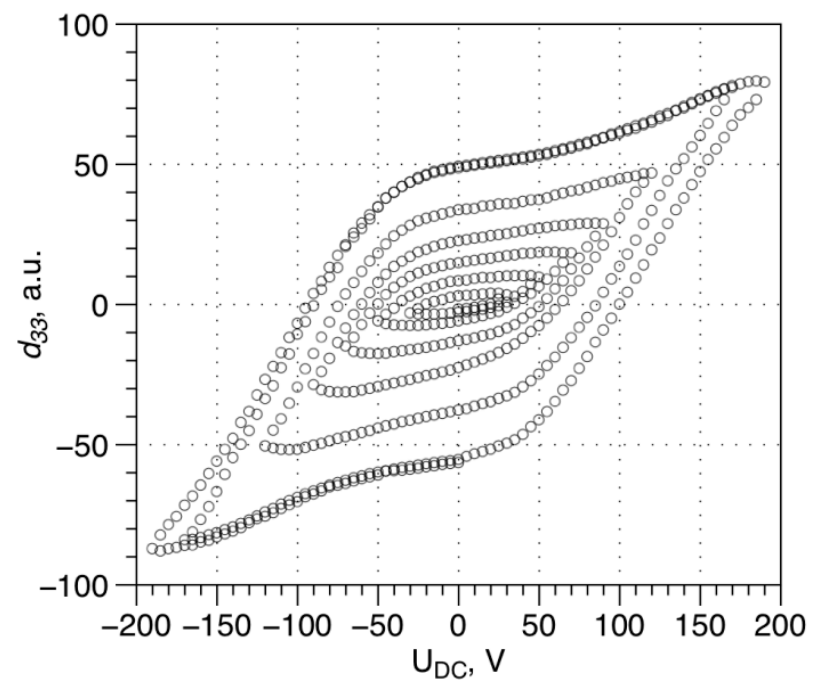

Fig. 5 Piezoresponse hysteresis obtained in a single as-electrospun BZT 1 fiber.

\section{Conclusions}

Evaluation of the first molecular hyperpolarizability $\beta$ of BZT 1 in a dioxane solution and thermal stability by TGA analysis revealed that BZT $\mathbf{1}$ is an efficient secondharmonic generator. Local piezoelectric measurements confirm that the heterocyclic system crystallizes in a nanocrystalline acentric structure when embedded in electrospun nanofibers. The local domain switching under an external electric field was studied by means of piezoresponse hysteresis loop acquisition. The observed out-of-plane hysteresis loop provides direct evidence for the switching of polarization in BZT 1 nanofibers and its ferroelectric properties.

We emphasize that even though it was not possible to obtain a viable bulk crystalline material, the use of the electrospinning technique allowed us to produce a nanocrystalline form of BZT 1 which clearly displays polar properties.

\section{Acknowledgements}

The authors wish to thank the Fundação para a Ciência e Tecnologia (FCT, Portugal) and FEDER-COMPETE for financial support through the Centro de Química and Centro de Física - Universidade do Minho, Projects PTDC/CTM/105597/2008 (FCOMP-01-0124-FEDER009457), PEst-C/QUI/UI0686/2013 (FCOMP-01-0124FEDER-037302) and a post-doctoral grant to R.M.F. Batista (SFRH/BPD/79333/2011). The NMR spectrometer Bruker Avance III 400 is part of the National NMR Network and was purchased within the framework of the National Program for Scientific Re-equipment, contract REDE/1517/RMN/2005 with funds from POCI 2010 (FEDER) and FCT.

\section{References}

Albert IDL, Marks TJ, Ratner MA (1997) Large molecular hyperpolarizabilities. quantitative analysis of aromaticity and auxiliary donor-acceptor effects. J Am Chem Soc 119:6575-6582. doi:10.1021/ja962968u

Balke N, Bdikin I, Kalinin S, Kholkin A (2009) Electromechanical imaging and spectroscopy of ferroelectric and piezoelectric materials: state of the art and prospects for the future. J Am Ceram Soc 92:1629-1647. doi:10.1111/j.1551-2916.2009.03240.x

Batista RMF, Costa SPG, Raposo MMM (2004) Synthesis of new fluorescent 2-(2',2 "-bithienyl)-1,3-benzothiazoles. Tetrahedron Lett 45:2825-2828. doi: 10.1016/j.tetlet.2004.02.048

Batista RMF, Costa SPG, Malheiro EL et al (2007) Synthesis and characterization of new thienylpyrrolyl-benzothiazoles as efficient and thermally stable nonlinear optical chromophores. Tetrahedron 63:4258-4265.

Breitung EM, Shu C-F, McMahon RJ (2000) Thiazole and thiophene analogues of donor-acceptor stilbenes: Molecular Hyperpolarizabilities and structure-property relationships. J Am Chem Soc 122:1154-1160. doi:10.1021/ja9930364

Coe BJ, Harris JA, Hall JJ et al (2006) Synthesis and quadratic nonlinear optical properties of salts containing benzothiazolium electronacceptor groups. Chem Mat 18: 5907-5918. doi:10.1021/cm061594t

Coelho P, Castro MCR, Fonseca AMC et al (2012a) Enhancement of the photochromic switching speed of bithiophene azo dyes. Tetrahedron Lett 53:4502-4506. doi:10.1016/j.tetlet.2012.05.166

Coelho P, Castro MCR, Fonseca AMC, Raposo MMM (2012b) Photoswitching in azo dyes bearing thienylpyrrole and benzothiazole heterocyclic systems. Dyes Pigments 92:745-748. doi:10.1016/j.dyepig.2011.06.019

Coelho P, Sousa CM, Castro MCR et al (2013) Fast thermal cis-trans isomerization of heterocyclic azo dyes in PMMA polymers. Opt Mater 35:1167-1172. doi:10.1016/j.optmat.2013.01.007

Costa SPG, Ferreira JA, Kirsch G, Oliveira-Campos AMF (1997) New fluorescent 1,3-benzothiazoles by the reaction of heterocyclic aldehydes with ortho-aminobenzenethiol. J Chem Res (S) 314315; (M) 2001-2013. doi:10.1039/A702605F

Costa SPG, Batista RMF, Cardoso P et al (2006) 2-arylthienyl-substituted 1,3-benzothiazoles as new nonlinear optical chromophores. Eur J Org Chem 3938-3946. doi:10.1002/ejoc.200600059

Cui Y, Qian G, Chen L, Wang Z, Gao J, Wang M (2006) Enhanced thermal stability of dipole alignment in inorganic-organic hybrid films containing benzothiazole chromophore. J Phys Chem B 110:4105-4110. doi:10.1021/jp057146a

Fu H-Y, Gao X-d, Zhong G-y et al (2009a) Synthesis and electroluminescence properties of benzothiazole derivatives. J Luminesc 129:1207-1214. doi:10.1016/j.jlumin.2009.06.004

Fu H-Y, Sun X-Y, Gao X-d et al (2009b) Synthesis and characterization of benzothiazole derivatives for blue electroluminescent devices. Synth Met 159:254 -259. doi:10.1016/j.synthmet.2008.09.013

Garcia-Amorós J, Castro MCR, Coelho P, Raposo MMM ,Velasco D (2013) New heterocyclic systems to afford microsecond green-light isomerisable azo dyes and their use as fast molecular photochromic switches. Chem Comm 49:11427-11429. doi: $10.1039 / \mathrm{C} 3 \mathrm{CC} 46736 \mathrm{H}$

Garcia-Amorós J, Reig M, Castro MCR, Cuadrado A, Raposo MMM, Velasco D (2014) Molecular photo-oscillators based on highly accelerated heterocyclic azo dyes in nematic liquid crystals. Chem Comm in press. doi: 10.1039/C4CC01450B

Hrobarik P, Hrobarikova V, Sigmundova I et al (2011) Benzothiazoles with tunable electron-withdrawing strength and reverse polarity: A route to triphenylamine-based chromophores with enhanced twophoton absorption. J Org Chem 76:8726-8736. doi:10.1021/jo201411t

Hrobárik P, Sigmundova I, Zahradník P et al (2010) Molecular engineering of benzothiazolium salts with large quadratic hyperpolarizabilities: Can auxiliary electron-withdrawing groups enhance nonlinear optical responses? J Phys Chem C 114:2228922302. doi:10.1021/jp108623d 
Hrobarik P, Zahradnik P, Fabian WMF (2004) Computational design of benzothiazole-derived push-pull dyes with high molecular quadratic hyperpolarizabilities. Phys Chem Chem Phys 6:495-502. doi:10.1039/B314150K

Hrobáriková V, Hrobárik P, Gajdoš P et al (2010) Benzothiazole-based fluorophores of donor- $\pi$-acceptor- $\pi$-donor type displaying high two-photon absorption. J Org Chem 75:3053-3068. doi: 10.1021/jo100359q

Isakov D, de Matos Gomes E, Belsley MS et al (2010a) High nonlinear optical anisotropy of urea nanofibers. Europhys Lett 91:28007. doi:10.1209/0295-5075/91/28007

Isakov DV, de Matos Gomes E, Almeida BG et al (2010b) Piezoresponse force microscopy studies of the triglycine sulfate-based nanofibers. J Appl Phys 108:042011-042014. doi:10.1063/1.3474966

Isakov DV, de Matos Gomes E, Vieira LG et al (2011a) Oriented singlecrystal-like molecular arrangement of optically nonlinear 2-methyl4-nitroaniline in electrospun nanofibers. ACS Nano 5:73-78 doi:10.1021/Nn101413x

Isakov D, de Matos Gomes E, Bdikin I et al (2011b) Production of polar $\beta$-glycine nanofibers with enhanced nonlinear optical and piezoelectric properties. Cryst Growth Des 11:4288-4291. doi: $10.1021 / \mathrm{cg} 2009336$

Isakov D, de Matos Gomes E, Belsley MS et al (2012) Strong enhancement of second harmonic generation in 2-methyl-4nitroaniline nanofibers. Nanoscale 4:4978-4982. doi:10.1039/C2NR30771E

Isakov DV, de Matos Gomes E, Almeida BG et al (2014) Energy harvesting from nanofibers of hybrid organic ferroelectric dabcoHReO . Apl $_{\text {Phy }}$ Lett 104:032907. doi:org/10.1063/1.4862437

Jin XF, Cui YJ, Gao Jk et al (2009) High thermally stable hybrid nonlinear optical films containing heterocycle chromophores. Thin Solid Films 517:5079-5083. doi:10.1016/j.tsf.2009.03.092

Kaatz P, Shelton DP (1996) Polarized hyper-Rayleigh light scattering measurements of nonlinear optical chromophores. J Chem Phys 105:3918-3929. doi:10.1063/1.472264

Kariduraganavar MY, Tambe SM, Tasaganva RG et al (2011) Studies on nonlinear optical polyurethanes containing heterocyclic chromophores. J Mol Struct 987: 158-165. doi:10.1016/j.molstruc.2010.12.010

Kuwabara J, Namekawa T, Haga M, Kanbara T (2012) Luminescent Ir(III) complexes containing benzothiazole-based tridentate ligands: synthesis, characterization, and application to organic light-emitting diodes. Dalton T 41:44-46. doi:10.1039/C1dt11560j

Li D, Wang Y, Xia Y (2003) Electrospinning of polymeric and ceramic nanofibers as uniaxially aligned arrays. Nano Lett 3:1167-1171. doi: $10.1021 / \mathrm{n} 10344256$

Li SPY, Tang TSM, Yiu KSM, Lo KKW (2012) Cyclometalated iridium(III)-polyamine complexes with intense and long-lived multicolor phosphorescence: synthesis, crystal structure, photophysical behavior, cellular uptake, and transfection properties. Chem Eur J 18:13342-13354. doi: 10.1002/chem.201200979

Liu Y, Guo H, Zhao J (2011) Ratiometric luminescent molecular oxygen sensors based on uni-luminophores of $\mathrm{CN}$ Pt(II)(acac) complexes that show intense visible-light absorption and balanced fluorescence/phosphorescence dual emission. Chem Comm 47:11471-11473. doi: 10.1039/C1CC14582G

Mabrouk A, Azazi A, Alimi K (2010) On the properties of new benzothiazole derivatives for organic light emitting diodes (OLEDs): A comprehensive theoretical study. J Phys Chem Solids 71:1225-1235. doi:10.1016/j.jpcs.2010.04.020

Miller RD, Lee VY, Moylan CR (1994) Substituted azole derivatives as nonlinear-optical chromophores. Chem Mat 6:1023-1032. doi: $10.1021 / \mathrm{Cm} 00043 \mathrm{a} 026$

Mishra A, Ma CQ, Bauerle P (2009) Functional oligothiophenes: Molecular design for multidimensional nanoarchitectures and their applications. Chem Rev 109:1141-1276. doi:10.1021/cr8004229

Morris JV, Mahaney MA, Huber JR (1976) Fluorescence quantum yield determinations. 9,10-Diphenylanthracene as a reference-standard in different solvents. J Phys Chem 80:969-974. doi: $10.1021 / \mathrm{j} 100550 \mathrm{a} 010$
Nye JF (2000) Physical properties of crystals: their representation by tensors and matrices. Clarendon Press, Oxford, UK.

Oudar JL (1977) Optical nonlinearities of conjugated molecules. Stilbene derivatives and highly polar aromatic compounds. J Chem Phys 67:446-457. doi:10.1063/1.434888

Oudar JL, Chemla DS (1977) Hyperpolarizabilities of the nitroanilines and their relations to the excited state dipole moment. J Chem Phys 66:2664-2668. doi:10.1063/1.434213

Persano L, Dagdeviren C, Su Y et al (2013) High performance piezoelectric devices based on aligned arrays of nanofibers of poly(vinylidenefluoride-co-trifluoroethylene). Nat Commun 4:1633. doi:10.1038/ncomms2639

Pina J, Seixas de Melo S, Batista RMF et al (2007) Spectral and photophysical characterization of donor-pi-acceptor arylthienyland bithienyl-benzothiazole derivatives in solution and solid state. Phys Chem A 111:8574-8578. doi:10.1021/Jp0730646

Pina J, Seixas de Melo S, Batista RMF et al (2010) The influence of the relative position of the thiophene and pyrrole rings in donoracceptor thienylpyrrolyl-benzothiazole derivatives. A photophysical and theoretical investigation. Phys Chem Chem Phys 12:9719-9725. doi:10.1039/C002434a

Raposo MMM, Castro MCR, Schellenberg P et al (2011a) Design, synthesis, and characterization of the electrochemical, nonlinear optical properties, and theoretical studies of novel thienylpyrrole azo dyes bearing benzothiazole acceptor groups. Tetrahedron 67:5189-5198. doi:10.1016/j.tet.2011.05.053

Raposo MMM, Castro MCR, Belsley M, Fonseca AMC (2011b) Push pull bithiophene azo-chromophores bearing thiazole and benzothiazole acceptor moieties: Synthesis and evaluation of their redox and nonlinear optical properties. Dyes Pigments 91:454-465. doi:10.1016/j.dyepig.2011.05.007

Raposo MMM, Fonseca AMC, Castro MCR et al (2011c) Synthesis and characterization of novel diazenes bearing pyrrole, thiophene and thiazole heterocycles as efficient photochromic and nonlinear optical (NLO) materials. Dyes Pigments 91:62-73. doi: 10.1016/j.dyepig.2011.02.012

Razus AC, Birzan L, Surugiu NM, et al (2007) Syntheses of azulen-1-ylbenzothiazol-2-yl diazenes. Dyes Pigments 74:26-33. doi:10.1016/j.dyepig.2006.01.041

Reis H (2006) Problems in the comparison of theoretical and experimental hyperpolarizabilities revisited. J Chem Phys 125:014506-014509. doi: 10.1063/1.2211611

Tao Y, Yang C, Qin J (2011) Organic host materials for phosphorescent organic light-emitting diodes. Chem Soc Rev 40:2943-2970. doi: 10.1039/C0CS00160K

Varanasi PR, Jen AK-Y, Chandrasekhar J et al (1996) The important role of heteroaromatics in the design of efficient second-order nonlinear optical molecules: theoretical investigation on push-pull heteroaromatic stilbenes. J Am Chem Soc 118:12443-12448. doi:10.1021/ja960136q

Wang RJ, Liu D, Ren HC et al (2011) Homoleptic tris-cyclometalated iridium complexes with 2-phenylbenzothiazole ligands for highly efficient orange OLEDs. Mater Chem 21:15494-15500. doi:10.1039/C1JM10757G

$\mathrm{Xu} \mathrm{XJ}$, Liao Y, Yu G et al (2007) Charge carrier transporting, photoluminescent, and electroluminescent properties of zinc(II)-2(2-hydroxyphenyl)benzothiazolate complex. Chem Mat 19:17401748. doi:10.1021/cm062960b

Zhang XH, Wong OY, Gao ZQ et al (2001) A new blue-emitting benzothiazole derivative for organic electroluminescent devices. Mater Sci Eng B-Solid State Mater Adv Technol 85:182-185. doi:10.1016/S0921-5107(01)00607-9

Zyss J, Oudar JL (1982) Structural dependence of nonlinear-optical properties of methyl-(2,4-dinitrophenyl)-aminopropanoate crystals. Phys Rev A 26:2016-2027. doi:10.1103/PhysRevA.26.2016 